\title{
Figure femminili di santità nella Commedia di Dante
}

Figures féminines de sainteté dans la Comédie de Dante

Holy Women in Dante's Comedy

\section{Giuseppe Ledda}

\section{CpenEdition}

\section{Journals}

\section{Edizione digitale}

URL: https://journals.openedition.org/cei/9640

DOI: $10.4000 /$ cei.9640

ISSN: 2260-779X

\section{Editore}

UGA Éditions/Université Grenoble Alpes

\section{Edizione cartacea}

ISBN: 978-2-37747-304-5

ISSN: $1770-9571$

Notizia bibliografica digitale

Giuseppe Ledda, «Figure femminili di santità nella Commedia di Dante», Cahiers d'études italiennes

[Online], 33 | 2021, online dal 01 octobre 2021, consultato il 13 octobre 2021. URL: http:// journals.openedition.org/cei/9640 ; DOl: https://doi.org/10.4000/cei.9640

Questo documento è stato generato automaticamente il 13 octobre 2021.

(c) ELLUG 


\title{
Figure femminili di santità nella Commedia di Dante
}

\author{
Figures féminines de sainteté dans la Comédie de Dante \\ Holy Women in Dante's Comedy
}

Giuseppe Ledda

1.

1 Nella poesia molteplice ed enciclopedica della Commedia, dove tutti i generi letterari sono presenti, inclusi e gerarchizzati nell'edificio summatico del poema, insieme a una pluralità di altri modelli, viene assunta, con modalità particolarmente rilevanti, anche l'agiografia, genere del discorso religioso che costituisce uno degli elementi fondamentali della cultura medievale. Il genere agiografico registra nel Duecento profonde innovazioni, grazie soprattutto all'azione degli ordini mendicanti. Si approntano nuovi repertori agiografici pensati come strumenti per il predicatore e insieme come raccolte di esempi di santità, mente il racconto della vita dei santi entra nella predicazione in modi nuovi. Alcuni dei repertori agiografici, fra cui la Legenda aurea di Iacopo da Varazze, hanno un'ampia diffusione in tutta Europa. Inoltre, si elaborano, non senza dispute e conflitti, le complesse tradizioni agiografiche relative ai nuovi santi, primi tra tutti i fondatori degli ordini mendicanti Francesco e Domenico ${ }^{1}$.

Questo aspetto così rilevante della cultura religiosa medievale trova nella Commedia uno spazio ampio e significativo. In particolare, la scrittura agiografica ha rilevo speciale nei canti del cielo del Sole, strutturati intorno al dittico agiografico dedicato ai santi fondatori degli ordini mendicanti, Francesco e Domenico. E la sequenza dei quattro cieli centrali si chiude nel settimo cielo, quello di Saturno, con un nuovo dittico agiografico, dedicato a due rappresentanti della santità monastica e contemplativa quali Pier Damiani e Benedetto da Norcia. Ma l'allusività al discorso agiografico è pervasiva e compare anche nell'Inferno, con modalità per lo più parodiche, e nel Purgatorio, soprattutto nell'ambito della retorica dell'esemplarità che è uno degli aspetti strutturali della cantica. 
3 Su alcuni di questi passaggi ho avuto modo di soffermarmi in altre sedi ${ }^{2}$. In questa occasione vorrei concentrarmi in particolare sulle figure di santità femminile, in cui si incrocia la grande attenzione del poeta per la ripresa di modelli e temi agiografici con la sensibilità ancora maggiore per le figure femminili e per la rappresentazione della donna ${ }^{3}$. L'intento è quello di offrire una prima rassegna delle figure di santità femminile, senza però la pretesa di dare in questa sede una trattazione approfondita di ognuna di esse ${ }^{4}$.

2.

4 Nell'Inferno, le allusioni all'agiografia sono soprattutto nell'ambito della parodia sacra, in quanto si applicano ai peccatori alcune pene che la tradizione agiografica riservava a certi martiri particolarmente venerati. Ma fin dal secondo canto appare l'importanza del tema mariano, che avrà un grande sviluppo in tutto il poema. L'iniziativa del soccorso celeste per il protagonista impedito dalle bestie e risospinto rovinosamente verso la selva si deve infatti a una «donna gentil» che «nel ciel [...] si compiange» dell'«impedimento» che ostacola e minaccia Dante, tanto da spezzare la severa sentenza divina (Inf. II, 94-96). Questa «donna gentile» non può che essere la Vergine Maria, il cui nome non compare mai nell'Inferno, come quello di Cristo. L'intervento della Vergine a favore degli uomini, anche prima che lo chiedano, sarà circolarmente ricordato nell'ultimo canto (Par. XXXIII, 16-18) e nel Paradiso il poeta parlerà anche della sua speciale devozione per Maria (XXIII, 88-89). La Vergine svolge il ruolo di intercessora per l'uomo presso Dio: il suo intervento misericordioso induce Dio a concedere il perdono. Tuttavia, occorre precisare che l'identificazione della «donna gentil nel ciel che si compiange» con la Vergine, oggi universalmente condivisa dai commentatori danteschi, è molto tarda e si deve al commentatore cinquecentesco Ludovico Castelvetro. Prima di allora, i commentatori antichi si limitavano a indicare il significato allegorico di questa donna, senza preoccuparsi della sua identità letterale ${ }^{5}$.

Nel corso del poema Maria avrà uno spazio fondamentale nel sistema degli esempi del Purgatorio. Le sette virtù che si contrappongono ai vizi capitali, cioè umiltà, amore misericordioso, mansuetudine, sollecitudine, distacco dai beni terreni, temperanza, castità, trovano in Maria la suprema realizzazione, tanto che in ogni cornice il primo esempio è sempre tratto dalla sua vita. Lo Speculum Beatae Mariae Virginis del francescano Corrado di Sassonia, ma circolato a lungo sotto il nome di Bonaventura da Bagnoregio, costituisce un importante precedente dell'operazione compiuta da Dante ${ }^{6}$. Infatti in questa opera si propone Maria come dotata al livello più alto delle sette virtù che si contrappongono ai sette vizi capitali. Maria è definita «contra superbiam profundissima per humilitatem, contra invidiam affectuosissima per caritatem, contra iram mansuetissima per lenitatem, contra acediam indefessissima per sedulitatem, contra avaritiam tenuissima per paupertatem, contra gulam temperatissima per sobrietatem, contra luxuriam castissima per virginitatem» (Lectio IV). E nel corso dell'opera Corrado propone esempi tratti dalla vita di Maria per ciascuna di queste virtù, esempi che in buona parte coincidono con quelli scelti da Dante. Fra il testo biblico e il riuso dantesco si pone talvolta un'importante opera di mediazione da parte della cultura religiosa medievale, che anche in questo caso gioca un ruolo rilevante, benché poi Dante attinga direttamente e autonomamente al testo evangelico. 
6 Nella prima cornice, dove le anime si purgano dal vizio della superbia, tra gli esempi di umiltà, scolpiti nella parte più bassa della parete del monte, il primo è l'episodio dell'Annunciazione: nel marmo è raffigurato l'arcangelo Gabriele che saluta Maria con la parola «Ave», che il lettore integra facilmente ricordando il testo evangelico («have gratia plena Dominus tecum benedicta tu in mulieribus» (Lc 1:28) ${ }^{7}$. Maria non insuperbisce per essere la prescelta, ma risponde semplicemente «Ecce ancilla Domini» (Lc 1:38), accettando umilmente la volontà divina (Purg. X, 34-45) ${ }^{8}$.

7 Nella cultura cristiana l'umiltà è la virtù che sola permette la vera ascesa, come afferma esplicitamente il detto evangelico secondo cui «qui autem se exaltaverit humiliabitur et qui se humiliaverit exaltabitur» (Mt 23:12). Per questo umiltà e altezza non sono in contraddizione, ma l'umiltà è la virtù che permette, abbassandosi, di ascendere alla vera altezza. Lo mostrerà in modo memorabile e definitivo ancora l'esempio di Maria, che al principio dell'ultimo canto del poema sarà ricordata come «umile e alta più che creatura» (Par. XXXIII, 2). Una particolare sottolineatura va sull'effetto salvifico dell'umiltà di Maria che accetta il decreto divino «de la molt'anni lagrimata pace» (Purg. X, 35), grazie a cui si riapre la strada fra la terra e il cielo, e l'uomo può, dopo un così lungo esilio, tornare a Dio.

Come esempio di amore misericordioso nella cornice dell'invidia viene proposto il comportamento di Maria alle nozze di Cana, il suo preoccuparsi non già per sé ma per gli altri. Quando si rese conto che il vino per la festa di nozze era terminato, lo segnalò al figlio, il quale con la trasformazione dell'acqua in vino operò il suo primo miracolo. In questo caso l'episodio è tratto dal Vangelo di Giovanni (Io 2:1-11) e si riprendono le parole stesse che Maria rivolge al figlio: «et deficiente vino dicit mater Iesu ad eum vinum non habent» (Io 2:3). In questa cornice gli esempi sono proclamati da una voce che passa velocissima nell'aria: «La prima voce che passò volando / 'Vinum non habent' altamente disse» (Purg. XIII, 25-30).

Maria è proposta come esempio di mansuetudine nella cornice dell'ira. In questo caso si tratta della sua mite reazione, «con atto / dolce di madre», al ritrovamento di Gesù fra $i$ dottori del Tempio, dopo una lunga e ansiosa ricerca (Purg. XV, 87-92). Qui Dante torna al modello di Luca, da cui traduce la battuta «Fili quid fecisti nobis sic ecce pater tuus et ego dolentes quaerebamus te» (Lc 2:48).

10 Nella cornice degli accidiosi sono proposti esempi di sollecitudine, il primo dei quali è ancora tratto dalla vita di Maria. Quando seppe dall'angelo che la sua parente Elisabetta era incinta nonostante la tarda età, si recò subito da lei, che abitava in una regione montana, per offrirle aiuto, nonostante lei stessa fosse a sua volta incinta. Le anime degli accidiosi purgano il loro «lento amore» correndo e gridando esempi, il primo dei quali è: «Maria corse con fretta a la montagna» (Purg. XVIII, 100). Anche in questo caso la traduzione dell'espressione di Luca è evidente: «abiit in montana cum festinatione» (LC 1:39).

11 Quale esempio mariano di povertà da contrapporre al vizio dell'avarizia Dante sceglie la maternità nella grotta di Betlemme: «Dolce Maria! [...] // Povera fosti tanto, / quanto veder si può per quello ospizio / dove sponesti il tuo portato santo» (Purg. XX, 19-24). Qui l'eco dal Vangelo è meno precisa ma risulta chiara l'allusione al racconto lucano (Lc 2:7).

12 Le nozze di Cana, già citate per l'esempio di carità, offrono anche quello di sobrietà alimentare da contrapporre al vizio della gola: «Più pensava Maria onde / fosser le 
nozze orrevoli e intere, / ch'a la sua bocca» (Purg. XXII, 142-144). Se nella seconda cornice si coglieva l'amore disinteressato che spinge Maria, qui si esalta il distacco dai propri bisogni di cui è segno il preoccuparsi degli altri e non di se stessa.

Infine Maria è esempio di castità nella cornice dei lussuriosi. Si riprende circolarmente l'episodio dell'Annunciazione che aveva offerto l'esempio iniziale (X, 34-45). $\mathrm{E}$ anche in questo caso si riporta direttamente una battuta del Vangelo di Luca, la risposta iniziale di Maria all'annuncio della concezione: «Virum non cognosco» (XXV, 128 e Lc 1:34).

Ma nel Purgatorio la presenza di Maria è insistita fin dai primi canti. Una possibile allusività mariana si potrà cogliere, forse non solo retrospettivamente, nell'emblema dello zaffiro, che apre la rappresentazione del paesaggio del nuovo regno, con il colore del cielo: «Dolce color d'orïental zaffiro» (Purg. I, 13). L'emblema dello zaffiro posto in apertura della cantica purgatoriale va collegato anche ai valori simbolici che i lapidari medievali assegnavano a questa pietra ${ }^{9}$. Tra questi spicca la virtù di liberare $i$ prigionieri dal carcere, aprire le porte serrate e sciogliere le catene, placare la divinità e renderla sensibile alle preghiere, come si legge nel Lapidario di Marbodo di Rennes: «educit carcere vinctos, / obstructasque fores et vincula tacta resolvit, / placatumque deum reddit, precibusque faventem $»^{10}$. L'unica altra occorrenza del termine zaffiro nel poema sarà nel canto XXIII del Paradiso, dove Maria appare incontro al pellegrino nel cielo delle stelle fisse e viene detta il «bel zaffiro / del quale il ciel più chiaro s'inzaffira» (vv. 101-102). Attraverso l'emblema dello zaffiro, Maria sembra rendersi presente già all'arrivo di Dante sulla spiaggia del Purgatorio, annunciando la sua importanza nella seconda cantica e prefigurando quella liberazione dalla prigionia del peccato che sarà compiuta e celebrata con la sua apparizione nel cielo delle Stelle fisse. Le virtù dello zaffiro possono ben essere accostate a quelle di Maria, che soccorre chi è imprigionato dal peccato e intercede presso Dio perché gli sia concessa la libertà.

Successivamente, nel primo riferimento esplicito, viene sottolineato il ruolo storico di Maria nella redenzione e nella rivelazione, proprio nel momento in cui Virgilio ricorda i limiti dell'umanità classica precristiana: «State contenti, umana gente, al quia; / ché, se potuto aveste veder tutto, / mestier non era parturir Maria» (Purg. III, 37-39). A sua volta Buonconte racconta della sua morte e del suo pentimento finale, con parole simili a quelle di Manfredi, ma con in più la menzione, straordinariamente significativa, del nome di Maria: «nel nome di Maria fini’» (Purg. V, 101).

16 Si conferma così l'importanza che la presenza mariana avrà nel regno purgatoriale. Poco più avanti, le anime nella «valletta» intonano la nuova preghiera del Salve, Regina (Purg. VII, 82), e Sordello segnala che i due angeli apparsi per respingere l'attacco della biscia diabolica «vegnon del grembo di Maria» (Purg. VIII, 37).

Nel Paradiso la presenza mariana è ovviamente insistita e importante. Una modalità rilevante di tale presenza si realizza tramite le preghiere mariane, con l'Ave Maria che incornicia la cantica e la visione dei beati (Par. III, 121-122 e XXXII, 95), sino poi alla spettacolare preghiera rivolta alla «Vergine Madre» da Bernardo (XXXIII, 1-39). Inoltre la presenza 'reale' di Maria ha grande spazio, dapprima nel cielo delle Stelle fisse (canto XXIII) e poi soprattutto nell'Empireo, attraverso la mediazione di un grande mariologo come Bernardo (canti XXXI-XXXIII). Lo svolgimento del tema mariano richiederebbe ovviamente un una trattazione esclusiva e assai ampia. Credo fosse però opportuno menzionarlo, sia pur rapidamente, nel quadro di una rassegna complessiva delle figure di santità femminile nel poema. 
3.

Nello stesso canto II dell'Inferno, accanto a Maria, e ovviamente a Beatrice ${ }^{11}$, appare anche un'altra santa a cui Dante si mostra particolarmente devoto: santa Lucia, che ha nel poema un ruolo sorprendentemente rilevante ${ }^{12}$. Dalla tradizione agiografica non sembrano emergere elementi decisivi per chiarire il senso di un tale rilievo: nella Legenda aurea, non emergono aspetti pertinenti in chiave dantesca, se non le consuete interpretationes nominis: «Lucia dicitur a luce»; «Vel dicitur Lucia quasi lucis uia» ${ }^{13}$. La figura di Lucia e il suo rapporto speciale con Dante sono piuttosto enigmatici. Ovviamente andrà tenuto presente il suo ruolo di protettrice degli occhi e della vista. Tuttavia, va ricordata anche l'ipotesi recentemente avanzata da Marco Santagata, il quale segnala che una chiesa dedicata a santa Lucia, Santa Lucia dei Magnoli, si trovava e si trova tuttora Oltrarno, lungo il tracciato urbano della via Cassia, proprio accanto alle case dei Bardi, dove presumibilmente doveva abitare Beatrice dopo il suo matrimonio con Simone de' Bardi. Ciò renderebbe probabile, secondo lo studioso, che in questa stessa chiesa fosse sepolta Beatrice dopo la sua morte ${ }^{14}$. Di qui si potrebbe ipotizzare un'intensa frequentazione di tale chiesa da parte di Dante e dunque una speciale familiarità del poeta con la figura di santa Lucia e la sua vicenda agiografica, che lì veniva ripetuta nella predicazione, nella liturgia, nell'iconografia. Si tratta come si vede di una serie di ipotesi suggestive ma prive di riscontri decisivi. La figura di Lucia nella Commedia sembra priva di ogni particolarità legata alla sua tradizione agiografica e pare attiva soprattutto nella sua funzione narrativa e simbolica.

In particolare, in Inferno II, Virgilio, per confortare Dante e convincerlo a iniziare il viaggio, racconta la discesa di Beatrice nel Limbo e l'antefatto celeste: l'iniziativa misericordiosa della Vergine Maria, la mediazione di Lucia, l'intervento diretto di Beatrice (Inf. II, 49-126). La Vergine, ottenuto con la propria misericordia il perdono divino, fa chiamare Lucia perché si muova al soccorso di Dante:

Donna è gentil nel ciel che si compiange

di questo impedimento ov' io ti mando,

sì che duro giudicio là sù frange.

Questi chiese Lucia in suo dimando

e disse: - Or ha bisogno il tuo fedele

di te, e io a te lo raccomando -. (Inf. II, 94-99)

Lucia sarebbe stata martirizzata, secondo la tradizione, il 13 dicembre 304, durante le persecuzioni di Diocleziano. Inoltre sarebbe stata accecata prima del martirio. Forse a causa di questo aspetto agiografico e del suo nome, collegato etimologicamente con lux, lucis, "luce", è considerata protettrice di coloro che soffrono di malattie agli occhi o alla vista (anche se non pare che tale prerogativa sia attestata in epoca dantesca o predantesca). Alcuni commentatori mettono in relazione la devozione di Dante con l'episodio raccontato nel Convivio secondo cui il poeta avrebbe sofferto di una malattia agli occhi (Conv. III, ix, 15). Tuttavia in tal caso si parla di una guarigione ottenuta con terapie mediche e non si fa cenno all'aiuto di Lucia. Va tenuta invece presente la connessione simbolica fra luce e grazia, specialmente grazia illuminante, e ciò spiega l'immediatezza con cui i commentatori antichi hanno inteso in tal senso Lucia.

Le parole della Vergine sono brevi e intense, fanno appello al bisogno che Dante ha dell'intervento di Lucia e lo indicano come il «fedele» della santa, a segnalare la sua 
speciale devozione ${ }^{15}$. Se la Vergine non si è mossa ma ha fatto chiamare Lucia, Lucia invece si muove, per recarsi da Beatrice:

Lucia, nimica di ciascun crudele,

si mosse, e venne al loco dov' i' era,

che mi sedea con l'antica Rachele. (Inf. II, 100-102)

Lucia va da Beatrice presso il suo seggio paradisiaco, la cui posizione sarà poi precisamente indicata quando Dante giungerà nell'Empireo (Par. XXXI, 67-69 e XXXII, 7-9), e anche lì Beatrice apparirà seduta accanto a Rachele. Lucia definisce Beatrice «loda di Dio vera», in quanto con la sua perfezione Beatrice costituisce una lode all'onnipotenza divina, capace di creare creature di tale bellezza fisica e morale, e chiede con modi concitati a Beatrice perché non abbia ancora soccorso Dante:

Disse: - Beatrice, loda di Dio vera,

ché non soccorri quei che t'amò tanto,

ch'uscì per te de la volgare schiera?

Non odi tu la pieta del suo pianto,

non vedi tu la morte che 'l combatte

su la fiumana ove 'l mar non ha vanto? -. (Inf. II, 103-108)

La santa incalza Beatrice con una serie di domande, tutte aperte dalla negazione non: domande retoriche che hanno il fine di esortare Beatrice all'azione ricordandole la terribile situazione in cui Dante si trova.

Più avanti nel corso del poema e del viaggio oltremondano, Lucia interviene direttamente per condurre Dante, immerso nel sonno, sino alla porta del Purgatorio. Addormentatosi nella valletta la sera precedente, quando la notte volge al termine e l'alba si avvicina, gli appare in sogno un'aquila dalle penne d'oro che cala su di lui, lo afferra e lo solleva in alto nel cielo sino a giungere alla sfera del fuoco, dove ha la sensazione di bruciare, tanto da risvegliarsi (Purg. IX, 19-30). Tra i tanti significati dell'aquila attivi nella cultura medievale ${ }^{16}$, uno dei più diffusi era quello legato al simbolismo penitenziale, sicuramente pertinente per un sogno che prefigura l'attraversamento penitenziale del Purgatorio, durante il quale anche Dante è coinvolto in alcune pene. Ma nella cultura medievale una serie di prerogative dell'aquila concorrono anche a costruire la simbologia del Cristo risorto: la regalità e la vittoria contro il serpente; la perenne giovinezza segno di resurrezione; l'altezza del volo che è simbolo dell'ascensione del Cristo risorto al cielo e del suo rapire verso l'alto anche l'anima del cristiano.

Nel momento in cui si risveglia e si ritrova in un luogo diverso da quello in cui si era addormentato Dante si mostra spaesato e impaurito. Così Virgilio, definito «il mio conforto» e «il mio signore», riprende subito la sua funzione di rassicurazione e di stimolo al viaggio, e offre i punti di riferimento spaziale che permettono a Dante (e al lettore) di orientarsi nello spazio, spiegando che i due sono giunti in prossimità del Purgatorio vero e proprio: «Tu se' ormai al purgatorio giunto: / vedi là il balzo che 'l chiude dintorno; / vedi l'entrata là 've par disgiunto» (Purg. IX, 49-51).

Tale situazione ricorda quella del secondo canto dell'Inferno, quando Dante aveva paura di compiere il viaggio nell'aldilà. In quel caso, Virgilio, per confortare Dante e convincerlo a iniziare il viaggio, racconta la discesa di Beatrice nel Limbo, e l'antefatto nel Paradiso. Analogamente, ora, il poeta latino spiega che mentre Dante dormiva, proprio Lucia, che era stata una delle «tre donne benedette» (Inf. II, 124), è stata protagonista di un intervento diretto. Infatti, spiega Virgilio: 
Dianzi, ne l'alba che procede al giorno, quando l'anima tua dentro dormia, sovra li fiori ond'è là giù addorno venne una donna, e disse: «I' son Lucia; lasciatemi pigliar costui che dorme;

sì l'agevolerò per la sua via». (Purg. IX, 52-57)

Come ricordavo, la figura di Lucia e il suo rapporto speciale con Dante sono piuttosto enigmatici. Qui naturalmente è importante il rapporto con l'aquila, anche se non vi può essere un appiattimento totale dei due elementi ${ }^{17}$, e Lucia sembra essere soprattutto un'immagine della grazia che scende sull'uomo e lo solleva verso il cielo. Inoltre appare evidente la sua relazione diretta con la Vergine, di cui la santa è una inviata, una intermediaria del suo soccorso misericordioso al fedele in difficoltà, aspetto ben presente fin dall'antefatto celeste di Inferno II.

Tale ruolo sarà ribadito del resto anche nella conclusione del poema. Nell'Empireo Lucia siede nell'ordine più alto e il suo seggio è di fronte a quello di Adamo, a sua volta accanto a Maria. Anche nell'Empireo dunque si ribadirà la posizione eminentissima di Lucia e il suo speciale rapporto con Maria. Lucia è l'ultimo dei beati a essere citato da san Bernardo: «siede Lucia, che mosse la tua donna / quando chinavi, a rovinar, le ciglia» (Par. XXXII, 137-138). Subito dopo aver visto Lucia, Dante viene invitato a spostare gli occhi verso la Vergine, mentre Bernardo intonerà la preghiera che questa conceda a Dante la visione di Dio. Dunque si torna da Lucia alla Vergine e dalla Vergine a Dio $^{18}$.

4.

Tra le donne citate nel IV canto dell'Inferno, spiccano quelle che si trovano nel «nobile castello» del Limbo e che sono connesse alla storia romana e alle origini troiane: la progenitrice dei Troiani Elettra, le guerriere «Cammilla e la Pantasilea» ${ }^{19}$, la figlia del re Latino e moglie di Enea Lavinia, le donne della Roma repubblicana più celebri per le loro virtù: «Lucrezia, Iulia, Marzïa e Corniglia» (Inf. IV, 121-128). Sapremo poi, nel primo canto del Purgatorio che qui si trova anche Marzia, la moglie di Catone (Purg. I, 78-90).

30 Ma nella prima parte del canto viene ricordato anche il descensus Christi ad Inferos e la salvazione delle anime dei 'padri' dal Limbo. Fra le anime condotte da Cristo con sé in Paradiso, viene citata anche una donna, Rachele, moglie di Giacobbe (Inf. IV, 60) ${ }^{20}$. Rachele era stata ricordata in Inf. II, 102 come vicina di Beatrice in Paradiso, e la sua posizione accanto a Beatrice nella disposizione dei beati nell'Empireo sarà ricordata ancora in Par. XXXII, 7-9, fra le donne beate nella candida rosa vissute prima di Cristo. Qui in Inferno IV, Rachele è l'unica donna citata nel breve catalogo delle anime liberate dal Limbo da parte di Cristo, il che conferma il rilievo eccezionale che tale figura ha nel poema, dove è significativamente sempre associata a Beatrice.

Inoltre le due sorelle mogli di Giacobbe, Lia e Rachele, erano interpretate dalla esegesi biblica come allegorie: la prima della vita attiva, la seconda della vita contemplativa. Forse in tal senso sono presenti nel terzo e ultimo sogno purgatoriale, in Purg. XXVII, 97-108, dove è probabile che prefigurino anche le due donne che Dante incontrerà nel 
giorno che sta iniziando: Lia alluderebbe figuralmente a Matelda e Rachele ancora a Beatrice, a cui è sempre associata nel poema.

5. il Santo Volto!» (XXI, 48), alludendo a un antico crocifisso in legno, ancora oggi custodito in una cappella della basilica di San Martino, che era oggetto di venerazione da parte dei lucchesi e che veniva riprodotto anche sulle monete di Lucca. La battuta ha evidentemente il significato 'Qui non siamo a Lucca', ma poiché i lucchesi erano soliti rivolgersi al Santo Volto per invocare aiuto, i diavoli vogliono dire al dannato che qui nessuno lo potrà più aiutare, nemmeno il Santo Volto. Del resto l'allusione alla religiosità dei lucchesi, che riprende quella relativa alla devozione a Santa Zita, è anche un altro modo per ricordare l'ipocrisia con cui i barattieri lucchesi si fingono devoti e pii, mentre in realtà si comportano da peccatori. Inoltre, il fatto che le monete lucchesi riportassero tale immagine potrebbe essere un'allusione al peccato del barattiere, e al fatto che qui non c'è più da guadagnare da traffici illeciti e corruzione ${ }^{23}$.

6.

Se nel Paradiso il discorso agiografico si dispiega pienamente, importanti anticipazioni del tema agiografico si manifestano già nel Purgatorio. La santità femminile ha qui una 
particolare importanza, attraverso il ruolo speciale attribuito a santa Lucia nel canto IX e soprattutto la fondamentale presenza mariana.

Inoltre si registra la presenza di episodi tratti dalla letteratura agiografica tra gli esempi di virtù presentati nelle cornici. In alcuni casi, le figure femminili sono coinvolte non come sante ma come le beneficiarie della santità maschile. L'esempio dell'umiltà di Traiano (Purg. X, 73-93), che coinvolge san Gregorio ${ }^{24} \mathrm{e}$ che sarà ricordato da Dante anche in Par. XX, 43-48 e 106-117, è molto diffuso in tutta la tradizione agiografica, ma anche in quella enciclopedica, nelle compilazioni dottrinali, nella letteratura esemplare. Qui naturalmente la figura di coprotagonista è la vedovella, con la sua umiltà e la sua forza nel richiedere la giustizia, mossa dal dolore e dall'amore per il figlio. Le espressioni dantesche enfatizzano questi caratteri: «e una vedovella li era al freno, / di lagrime atteggiata e di dolore» (Purg. X, 77-78); «La miserella [...] / pareva dir: "Segnor, fammi vendetta / di mio figliuol ch'è morto, ond'io m'accoro» (82-84); «come persona in cui dolor s'affretta» (87); «la vedovella consolò del figlio» (Par. XX, 45).

Alla tradizione agiografica risale anche l'esempio di povertà, o meglio di «larghezza», da parte di san Nicola:

Esso parlava ancor de la larghezza

che fece Niccolò a le pulcelle,

per condurre ad onor lor giovinezza. (Purg. XX, 31-33)

Si tratta di una notizia diffusissima e proposta in apertura del capitolo su Nicola nella Legenda aurea ${ }^{25}$. In realtà, la tradizione agiografica attribuisce la "povertà» al padre delle tre fanciulle, non a Nicola, per il quale anzi si indica l'origine da una ricca famiglia. Così nella Legenda aurea si dice che Nicola «ex diuitibus et sanctis parentibus originem duxit ${ }^{26}$, mentre al padre delle ragazze si attribuisce la povertà, a causa della quale si sentiva costretto a prostituire le figlie: «conuicaneus suus satis nobilis tres filias uirgines ob inopiam prostituere cogitur» ${ }^{27}$. Si ricordano poi una serie di virtù di cui Nicola dà prova una volta eletto vescovo, ma senza traccia di 'povertà'. Del resto, mentre $\mathrm{i}$ primi due esempi citati in Purgatorio XX, Maria e Fabrizio, sono esplicitamente esempi di povertà (v. 22: «povera fosti tanto»; v. 26: «con povertà volesti anzi virtute»), quella di Nicola è piuttosto una prova di liberalità («larghezza»): egli è un esempio della scelta di usare le ricchezze non per ottenere vane lodi umane e terrene ma per la gloria di Dio. Le ragazze beneficiate, «le pulcelle», hanno un certo spazio nel pur breve accenno dantesco, in quanto il gesto di Nicola ha il fine di «condurre ad onor lor giovinezza», attraverso il matrimonio.

Colpisce però la totale assenza di figure di santità femminile proprie della tradizione agiografica cristiana nella serie degli esempi di virtù. Le uniche figure femminili esemplari qui proposte, oltre ovviamente a Maria, sono infatti «le Romane antiche», citate fra gli esempi di temperanza nella cornice dei golosi, in quanto "per lor bere contente furon d'acqua» (Purg. XXII, 145-146), e la dea Diana, posta fra gli esempi di castità nella cornice dei lussuriosi, per la sua inflessibile severità nei confronti della ninfa Elice che aveva ceduto alla violenza di Giove perdendo la verginità. In questa stessa serie si trova anche l'unico esempio 'generico', quello dello spirito virtuoso e casto con cui mogli e mariti vivono la sessualità nel matrimonio: «indi donne / cantavano e mariti che fuor casti / come virtute e matrimonio imponne» (Purg. XXV, 133-135). 

guelfa gli attribuiva i peggiori peccati, Manfredi doveva essere ovviamente ritenuto da tutti dannato all'Inferno. Invece è la prima anima che Dante incontra fra quelle del Purgatorio, destinate alla salvezza. Perciò la richiesta di informare la figlia Costanza sembra inizialmente avere questo fine consolatorio. Tuttavia, nell'ultima parte del suo discorso, Manfredi spiega che a causa della morte in stato di scomunica, pur perdonato e salvato da Dio, egli deve però attendere «da questa ripa in fore», cioè fuori della parete rocciosa che delimita il Purgatorio vero e proprio, per un periodo pari a trenta volte il tempo che ha vissuto nel suo stato di ribellione alla Chiesa, «se tal decreto / più corto per buon prieghi non diventa» (Purg. III, 136-141). L'aggiunta finale introduce il tema delle preghiere di suffragio, che viene subito sviluppato: 
«Vedi oggimai se tu mi puoi far lieto,

revelando a la mia buona Costanza

come m'hai visto, e anco esto divieto;

ché qui per quei di là molto s'avanza». (Purg. III, 142-145)

Se già all'inizio del suo discorso Manfredi aveva invitato Dante a recarsi dalla figlia Costanza, ora aggiunge la richiesta delle preghiere: infatti il tempo di attesa decretato per le anime può diventare più breve grazie alle preghiere dei buoni ancora viventi. È un principio fondamentale nella struttura del Purgatorio. Il pentimento del peccatore deve essere seguito da un pieno processo penitenziale: la confessione, l'assoluzione, gli atti di riparazione e di carità imposti dal confessore. Nel momento del pentimento sincero, anche al di fuori del sacramento confessionale, Dio accoglie la richiesta di perdono e cancella la colpa del peccato. Resta però la pena, come un debito che deve essere pagato, in parte attraverso le penitenze e le opere buone in vita, in parte attraverso le pene purificatorie del Purgatorio dopo la morte. Qui, sia nell'Antipurgatorio sia nel Purgatorio vero e proprio, il debito che le anime devono pagare è in termini di tempo: un tempo di attesa nell'Antipurgatorio e un tempo di purgazione nel Purgatorio. Ma i vivi possono aiutare le anime a pagare questo debito offrendo per loro suffragi, messe, preghiere, atti di penitenza e di carità. Infatti, in virtù del principio della comunione dei santi, i beni spirituali possono essere condivisi fra tutti i cristiani, vivi e defunti. Per questo tutte le anime chiederanno a Dante di ricordare ai loro cari di pregare per $\operatorname{loro}^{28} \mathrm{e}$ in vari modi il tema delle preghiere di suffragio avrà numerose riprese e sviluppi nei canti successivi e lungo tutta la cantica ${ }^{29}$.

In alcuni di questi casi, ha un'enfasi particolare il riferimento alle figure femminili, le cui preghiere e i cui pianti penitenziali offerti a suffragio dei cari defunti hanno uno straordinario valore salvifico ${ }^{30}$. Cito solo qualche altro caso a titolo esemplificativo: Giovanna, figlia di Nino Visconti (Purg. VIII, 70-72: «Quando sarai di là da le grandi onde, / dì a Giovanna mia che per me chiami / là dove a li 'nnocenti si risponde»); Alagia dei Fieschi, nipote del papa Adriano V (XIX, 142-145: «Nepote ho io di là c'ha nome Alagia, / buona da sé, pur che la nostra casa / non faccia lei per essempro malvagia; // e questa sola m'è di là rimasa»); Nella, moglie di Forese Donati (XXIII, 85-93: «Sì tosto m'ha condotto / a ber lo dolce assenzo d'i martìri / la Nella mia con suo pianger dirotto.// Con prieghi devoti e con sospiri / tratto m'ha de la costa ove s'aspetta, / e liberato m'ha de li altri giri. // Tanto è a Dio più cara e più diletta / la vedovella mia, che molto amai, / quant'ella è più soletta»).

Talvolta, accanto alle donne in preghiera, implicitamente proposte come esempi virtuosi per i lettori e le lettrici del poema, si delineano invece le figure negative di donne che trascurano di pregare per i propri cari, come la moglie di Nino Visconti, Beatrice d'Este, contrapposta alla figlia Giovanna (Purg. VIII, 73-81). Ma già nei primi canti, dopo l'esempio edificante di Costanza, figlia di Manfredi, veniva ricordata negativamente Giovanna, la moglie di Buonconte (VI, 89-90: «Giovanna o altri non hanno di me cura; / perch'io vo tra costor con bassa fronte»). Ad Alagia si contrappone invece, nelle parole di papa Adriano, il resto della famiglia, la «casa», che minaccia di rendere anche «lei per essempro malvagia» (XIX, 143-144). L'esempio pio e salvifico di Nella si contrappone poi a quello delle «svergognate donne fiorentine» con i loro costumi degenerati e impudichi (XXIII, 91-111).

Prima di lasciare il Purgatorio, posso fare solo un rapido cenno a una delle figure più enigmatiche della Commedia, quella di Matelda, oggetto di numerose e controverse interpretazioni, e in qualche caso messa in relazione con sante e mistiche di nome 
Matilde $^{31}$. Osservo solo che nel suo caso non viene mai usato il lessico della santità $\mathrm{e}$ pare debole e vaga anche la perifrasi «quella pia / [...] che conducitrice / fu de' miei passi lungo 'l fiume pria» (Purg. XXXII, 82-84). Spicca invece soprattutto il lessico della bellezza, tanto che «la bella donna» è il sintagma con cui viene indicata per ben quattro volte (Purg. XXVIII, 43 e 148; XXXI, 100; XXXIII, 121).

8.

49 Nel Paradiso, la ripresa della scrittura agiografica, prima di acquisire una nuova funzione strutturale nei canti del cielo del Sole, è evidente fin dal primo incontro con le anime beate nel cielo della Luna. Qui si registra infatti la prima esplicita menzione nella cantica della figura di un santo celebrato dalla tradizione agiografica, e si tratta di una figura femminile. Le anime qui incontrate, Piccarda Donati e Costanza d'Altavilla, sono due figure di rilievo e a loro modo esemplari, pur nella imperfezione della loro condotta terrena $^{32}$. Proprio per tale imperfezione, e in particolare per il loro venir meno ai voti presi, sono beate ma «ne la spera più tarda» (Par. III, 51). Ma tale imperfezione non pregiudica per loro la possibilità di salvezza, grazie alla fedeltà, almeno interiore, che hanno serbato nei confronti del modello perfetto di santa Chiara, del cui ordine Piccarda è stata monaca. Chiara è così la prima santa esaltata come beata nei gradi più alti della gloria celeste:

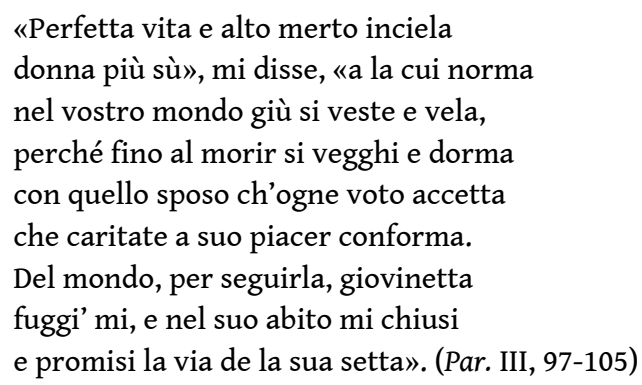

Non ha naturalmente molto senso interrogarsi sul cielo "più sù» in cui Chiara si troverebbe ${ }^{33}$. Qualcuno ha comunque ipotizzato che potrebbe trattarsi del cielo di Saturno, dei contemplanti ${ }^{34}$. Indubbiamente è ipotesi sensata e probabile, tuttavia mancano indizi precisi in tal senso. Qui tale collocazione superiore come effetto di una vita perfetta serve soprattutto a opporsi a quella inferiore riservata alle due monache seguaci imperfette e manchevoli di Chiara.

In ogni caso, è certamente importante la volontà dantesca di mettere rilievo santa Chiara, la prima figura di caratura agiografica di cui si parla nella terza cantica ${ }^{35}$. Per le terzine su Chiara sono state chiamate in causa alcune fonti agiografiche, tra cui la Vita Francisci di Tommaso da Celano:

Hic est locus ille beatus et sanctus, in quo gloriosa religio et excellentissimus Ordo Pauperum Dominarum et sanctarum uirginum, a conuersione beati Francisci fere sex annorum spatio iam elapso, per eundem beatum uirum felix exordium sumpsit; in quo domina Clara, ciuitate Assisii oriunda, lapis pretiosissimus atque fortissimus caeterorum superpositorum lapidum exsistit fundamentum. Nam, cum post initiationem Ordinis fratrum dicta domina sancti uiri monitis ad Deum conuersa fuisset, multis exstitit ad profectum innumeris ad exemplum. Nobilis parentela sed nobilior gratia; uirgo carne, mente castissima; aetate iuvencula sed animo cana; constans proposito et diuino amore amore ardentissima desiderio; sapientia praedita et humilitate praecipua: Clara nomine, uita clarior, clarissima moribus ${ }^{36}$. 
Nelle righe di Tommaso da Celano su Chiara è particolarmente rilevante, in relazione al passo dantesco, l'insistenza sulla verginità e castità («uirgo carne, mente castissima») e la costanza nel mantenere i propri propositi («constans proposito»), soprattutto in contrasto con il venir meno ai voti, e in particolare proprio a quello di castità, da parte di Piccarda e di Costanza. Sono aspetti ancora ripetuti dal Celanese anche in riferimento alle seguaci e compagne di Chiara:

Tertio vuirginitatis et castitatis lilium sic respergit odore mirabilia universas ut, terrenarum cogitationum oblitae, sola desiderant caelestia meditari, et tantus ex ipsius fragrantia in ipsarum cordibus aeterni Sponsi amor exoritur, ut integritas sacrae affectionis omnem ab eis consuetudinem vitae prioris excludat ${ }^{37}$.

Qui, oltre ai temi già segnalati, ha rilievo anche la metafora nuziale («in ipsarum cordibus aeterni Sponsi amor exoritur»), un aspetto che è fortemente valorizzato anche nel testo dantesco. Infatti, è qui che per la prima volta troviamo nel Paradiso la metafora delle nozze mistiche, che tanto spazio avrà nel corso della cantica e specie in quei canti in cui è più chiara ed intensa la dimensione agiografica. Nel discorso di Piccarda le nozze a cui si fa riferimento sono quelle con Cristo, «quello sposo ch'ogne voto accetta / che caritate a suo piacer conforma» (Par. III, 101-102) ${ }^{38}$.

Altrettanto interessanti le consonanze con alcune espressioni della Legenda Maior di Bonaventura:

Conuertebantur etiam uirgines ad perpetuum coelibatum, inter quas uirgo Deo carissima Clara, ipsarum plantula prima, tamquam flos uernans et candidus odorem dedit et tamquam stella perfulgida radiauit. Haec nunc glorificata in caelis, ab Ecclesia digne venerantur in terris, quae filia fuit in Christo sancti patris Francisci et mater Pauperum Dominarum ${ }^{39}$.

Transeuntes quoque per ecclesiam Sancti Damiani, in qua uirgo illa nobilis Clara, nunc gloriosa in caelis, tunc inclusa cum uirginibus morabatur ${ }^{40}$.

Di rilievo anche qui l'enfasi sulla verginità, sulla conversione al celibato e alla castità eterna da parte di Chiara e delle sue compagne («Conuertebantur etiam uirgines ad perpetuum coelibatum»), proprio quegli aspetti violati da Piccarda. E ancora l'enfasi sulla attuale beatitudine di Chiara nella gloria celeste («nunc glorificata in caelis»; «nunc gloriosa in caelis»). Secondo alcuni studiosi, dal sintagma «in caelis», ripetuto nel testo di Bonaventura, potrebbe anche derivare il neologismo dantesco «inciela» ${ }^{41}$.

9.

Dopo l'incontro con le anime beate, ma «ne la spera più tarda», di Piccarda e Costanza d'Altavilla (che porta all'allusione all'esempio luminoso di santa Chiara), nei vari cieli del Paradiso, Dante non incontrerà nessuna figura femminile, se non Cunizza e Raab, entrambe nel cielo di Venere, nel corso di un episodio a proposito nel quale, più che d'amore o di santità si parla soprattutto di politica ${ }^{42}$.

In particolare, di Cunizza si ricordano i peccaminosi trascorsi giovanili preda dell'amore folle (Par. IX, 32-33: «qui refulgo / perché mi vinse il lume d'esta stella»), e si allude velatamente alla conversione (IX, 34-36: «ma lietamente a me medesma indulgo / la cagion di mia sorte, e non mi noia; / che parria forse forte al vostro vulgo»), ma non si indicano particolari prove di santità, mentre le si affidano piuttosto una serie di discorsi profetici di forte impronta politica ${ }^{43}$. 
Di Raab si esalta il ruolo giocato nella conquista della Terra santa da parte di Giosuè e l'assunzione al cielo da parte di Cristo («Da questo cielo [...] pria ch'altr'alma / del triunfo di Cristo fu assunta», Par. IX, 118-120). Sono stati chiamati in causa dagli studiosi i vari simbolismi attribuiti a Raab dall'esegesi biblica, in cui è solitamente considerata figura della Chiesa, con diverse sfumature. In ogni caso, nel riferimento dantesco non sembra assumere un profilo particolare come modello di santità ${ }^{44}$.

Dopo queste quattro beate incontrate nel primo e nel terzo cielo, dove ancora giunge il cono d'ombra proiettato dalla Terra (Par. IX, 118-119) e dove si fanno incontro a Dante beati che hanno qualche ombra nella loro condotta terrena, non si registrano presenze femminili nei quattro cieli centrali, Sole, Marte, Giove e Saturno, dove si manifestano a Dante i beati che hanno praticato al livello più alto, nella vita cristiana, le quattro virtù cardinali. Nel cielo delle Stelle fisse, nel trionfo di Cristo, scende incontro a Dante anche Maria col suo corpo glorioso.

Ma per trovare altre donne beate del Paradiso, di là da Beatrice e Maria, bisogna arrivare all'Empireo, che appare come una rosa celeste e come un immenso anfiteatro. Come spiega a Dante san Bernardo nel canto XXXII, esso presenta da una parte coloro che sono vissuti prima di Cristo e hanno creduto alla sua venuta futura, dall'altra coloro che sono vissuti dopo Cristo: questa seconda metà naturalmente presenta ancora qualche posto vuoto. Una delle due linee verticali divisorie tra le due metà dell'anfiteatro è segnata da Maria, sotto la quale stanno una serie di grandi donne del mondo precristiano di cui narra l'Antico Testamento: Eva, Rachele (accanto alla quale si trova, sullo stesso livello, Beatrice), Sara, Rebecca, Giuditta e Ruth. La linea che si trova esattamente di fronte a quella sul cui punto più alto siede Maria è costituita invece da Giovanni Battista, posto sul grado più alto e quindi perfettamente di fronte a Maria, sotto il quale si trovano i fondatori di grandi esperienze e regole monastiche: Francesco, Benedetto e Agostino. La mirabile provvidenza divina ha previsto che ognuna delle due metà del paradiso avrà alla fine dei tempi lo stesso numero di beati.

1 I due che siedono accanto a Maria, da una parte e dall'altra, «son d'esta rosa quasi due radici» (v.120): Adamo a sinistra e san Pietro a destra. Accanto a Pietro siede san Giovanni evangelista, autore anche dell'Apocalisse, mentre accanto ad Adamo si trova Mosè. Di fronte a Pietro, quindi sulla destra di Giovanni Battista (che è di fronte a Maria) siede sant'Anna, la madre di Maria. Di fronte ad Adamo, quindi alla sinistra del Battista, siede santa Lucia, che ha dunque una posizione straordinariamente elevata e importante anche nell'Empireo.

Rispetto alla totale assenza di figure femminili nei quattro cieli centrali del Paradiso, nell'Empireo la presenza di donne è quindi ampia e significativa. Oltre Maria sono registrate Eva, Rachele, Sara, Rebecca, Giuditta e Ruth; poi sant'Anna e santa Lucia, oltre ovviamente a Beatrice. Sono tutte figure bibliche, mentre l'unica santa postbiblica dei tempi cristiani, a parte Beatrice, è santa Lucia. Si conferma così, conclusivamente, il rilievo eccezionale assegnato da Dante a questa figura.

3 E, benché non citata qui ma in apertura della cantica, assume un rilevo ancora maggiore la figura di santa Chiara, che resta l'unica santa moderna ad avere l'onore di un riferimento tanto ampio ed enfatico come quello del canto III, un riferimento costruito sui modelli più alti del discorso agiografico, quasi ad anticipare lo sviluppo di tali registri nei canti del cielo del Sole. 
64 trattazione dantesca delle donne, che si esprime anche nella rappresentazione delle
figure femminili di santità. Queste figure non sono in realtà particolarmente numerose,
ma svolgono una funzione strutturale estremamente forte e significativa.

\section{NOTE}

1. Naturalmente la bibliografia sull'agiografia medievale è sterminata. Segnalo solo qualche lavoro complessivo di particolare importanza e utilità: A. Vauchez, La santità nel medioevo, Bologna, Il Mulino, 1989 (1981); C. Leonardi, Agiografie medievali, Firenze, SISMEL, 2011. In particolare, per quanto riguarda Francesco e l'agiografia francescana, mi limito a rimandare, anche per ulteriori riferimenti bibliografici, ai volumi della serie La letteratura francescana, a cura di C. Leonardi, con la collaborazione di D. Solvi, Milano, Fondazione Lorenzo Valla-Mondadori, 6 voll., 2004 ss. Su Domenico si veda almeno H. Vicaire, Storia di san Domenico, Nuova edizione italiana a cura di Valerio Ferrua o.p., Cinisello Balsamo (Milano), Edizioni Paoline, 1987; L. Canetti, «Domini custos». Contributi alla storia di san Domenico nelle fonti agiografiche del XIII secolo, Sala Baganza (Parma), Editoria Tipolitotecnica, 1994; Id., L'invenzione della memoria. Il culto $e$ l'immagine di Domenico nella storia dei primi frati Predicatori, Spoleto, Centro italiano di studi sull'alto medioevo, 1996. Sulla Legenda aurea, A. Boureau, La Légende dorée. Le système narratif de Jacques de Voragine (†1298), Paris, Éditions du Cerf, 1984; G. P. Maggioni, Ricerche sulla composizione e sulla trasmissione della «Legenda aurea», Spoleto, Centro italiano di studi sull'alto medioevo, 1995.

2. Cfr. G. Ledda, Osservazioni sul panegirico di San Domenico («Par.»XII, 31-114), «L'Alighieri», a. XLVII, n.s. 27, 2006, pp. 105-125; Id., San Pier Damiano nel cielo di Saturno, «L'Alighieri», a. XLIX, n.s. 32, 2008, pp. 49-72; Id., Agiografia e autoagiografia nel «Paradiso», in «Atti dell'Accademia di Scienze Arti e Lettere di Modena. Memorie Scientifiche, Giuridiche, Letterarie», Serie VIII, vol. XVIII (2015), fasc. I., pp. 309-333; Id., Poesia e agiografia nella «Commedia», in G. Ledda (a cura di), Dante poeta cristiano e la cultura religiosa medievale. In ricordo di Anna Maria Chiavacci Leonardi, Ravenna, Centro Dantesco dei Frati Minori Conventuali, 2018, pp. 215-258.

3. Su questo tema più generale, oltre ai saggi inclusi nel presente fascicolo, rimando, anche per ulteriori riferimenti bibliografici, a due libri recenti: M. Santagata, Le donne di Dante, Bologna, Il Mulino, 2021; E. Lombardi, Beatrice e le altre. Dante e le figure femminili, Roma, GEDILa Repubblica, 2021. In entrambi la dimensione della santità femminile e il rapporto con l'agiografia viene però sostanzialmente ignorato. Nel secondo si trova un capitolo dal titolo «Sante e prostitute», ma le uniche figure citate per il primo aspetto sono quelle bibliche: Maria, Eva e Raab. Qualche osservazione, limitatamente alle figure di provenienza biblica, in R. Mercuri, Figure femminili bibliche nella «Commedia» di Dante, «Linguistica e Letteratura», vol. XLI, n ${ }^{\circ} 1-2$, 2016, pp. 61-69.

4. Le strutture del racconto agiografico sono state riconosciute già nella Vita nova. In uno studio importante e seminale, Vittore Branca mostrò l'importanza dei modelli dell'agiografia femminile duecentesca per la costruzione del personaggio di Beatrice e per le modalità del discorso vitanovesco sulla gentilissima. Cfr. V. Branca, Poetica del rinnovamento e tradizione agiografica nella «Vita Nuova», «Letture classensi», $n^{\circ} 2,1969$, pp. 31-66. Altri studiosi hanno invece enfatizzato l'intertestualità evangelica e gli elementi cristologici relativi alla rappresentazione di Beatrice. Cfr. per esempio C.S. Singleton, An Essay on the "Vita Nuova», Cambridge (Mass.), Harvard 
University Press, 1949; G. Gorni, La Beatrice di Dante, dal tempo all'eterno, in Dante Alighieri, Vita Nova, a cura di L. C. Rossi, intr. di G. Gorni, Milano, Mondadori, 1999, pp. V-XL.

5. Cfr. in proposito C. Santarelli, Per l'interpretazione delle «donne benedette» («Inferno»II), «L'Alighieri», a. LXI, n.s. 56, 2020, pp. 107-129.

6. Per il testo, cfr. Corrado di Sassonia, Speculum seu Salutatio Beatae Mariae Virginis ac Sermones, a cura di P. de Alcantara Martinez, Roma, Editiones Collegii S. Bonaventurae ad Claras Aquas, 1975. Sul rapporto con gli exempla mariani nel Purgatorio cfr. S. Girotto, L'influsso dello Speculum B. M. Virginis nella Divina Commedia, in Corrado di Sassonia. Predicatore e mariologo del sec. XIII, Firenze, Biblioteca di Studi Francescani, 1952, pp. 202-216; F. Mancini, Un'«Auctoritas» di Dante, «Studi Danteschi», vol. XLV, 1968, pp. 95-119; M. Semola, Maria e gli altri «exempla» biblici nei canti X-XXVI del «Purgatorio», in Memoria biblica e letteratura italiana, Napoli, Istituto Universitario Orientale, 1998, pp. 9-32; C. Crevenna, Strategie ricorsive negli exempla del «Purgatorio» dantesco, «ACME», vol. LVII, 2004, pp. 33-54; M. Boccuti, «Quella ch'ad aprir l'alto amor volse la chiave»: Maria «Domina Dei» tra patrimonio laudistico e innovazione, «Rivista di Letteratura Religiosa Italiana», vol. I, 2018, pp. 13-24. Sul ruolo di Maria nel Purgatorio, cfr. anche A. M. Chiavacci Leonardi, Le beatitudini e la struttura poetica del «Purgatorio», "Giornale storico della letteratura italiana», vol. CLXI, n ${ }^{\circ} 513$, 1984, pp. 1-29, a pp. 26-29.

7. I passi biblici sono citati da Biblia Sacra iuxta Vulgatam Versionem, a cura di R. Weber et al., terza ed., a cura di B. Fischer et al., Stuttgart, Deutsche Bibelgesellschaft, 1983 (da tale edizione sono tratte anche le abbreviazioni usate).

8. Si tratta appunto di uno dei casi in cui la scelta dantesca dell'episodio mariano coincide con quella di Corrado di Sassonia. Cfr. M. Semola, Maria e gli altri «exempla» biblici nei canti X-XXVI del «Purgatorio» dantesco, cit.

9. Cfr. in proposito Ezio Raimondi, «Rito e storia nel i canto del Purgatorio», in Id., Metafora e storia. Studi su Dante e Patrarca, Torino, Einaudi, 1970, pp. 65-94, a pp. 69-70.

10. Marbodo di Rennes, De lapidibus, vv. 116-118 (in Lapidari. La magia delle pietre preziose, a cura di Bruno Basile, Roma, Carocci, 2006, p. 46).

11. La bibliografia su Beatrice è ovviamente sterminata. Mi limito a rimandare a due volumi miscellanei: M. Picchio Simonelli (a cura di), Beatrice nell'opera di Dante e nella memoria europea, 1290-1990, Atti del Convegno internazionale (10-14 dicembre 1990), Firenze / Napoli, Cadmo / Istituto Universitario Orientale, 1994; R. Abardo (a cura di), Omaggio a Beatrice (1290-1990), Firenze, Le Lettere, 1997.

12. La figura di Lucia e il suo rapporto speciale con Dante sono piuttosto enigmatici. Per alcune ipotesi, cfr. A. K. Cassell, Santa Lucia as Patroness of Sight: Hagiography, Iconography, and Dante, «Dante Studies», $\mathrm{n}^{\circ}$ 109, 1991, pp. 71-88; F. Spera, Il dogmatismo dantesco e il lettore moderno, in B. Peroni (a cura di), Leggere e rileggere la «Commedia» dantesca, Milano, Unicopli, 2009, pp. 17-35, alle pp. $18-27$.

13. Iacopo da Varazze, Legenda aurea, ed. critica a cura di P. Maggioni, Firenze, SISMEL-Edizioni del Galluzzo, 1998, cap. IV, par. 1 e cap. IV, par. 1 (p. 49).

14. M. Santagata, Dante. Il romanzo della sua vita, Milano, Mondadori, 2012, pp. 40-44. Cfr. ora anche Id., Le donne di Dante, cit., pp. 85-86.

15. Degno di nota che lo stesso termine sarà usato per san Bernardo, «fedele» della Vergine, in Par. XXXI, 102.

16. Per una discussione più ampia di questa parte e per ulteriori riferimenti bibliografici, cfr. G. Ledda, Il bestiario dell'aldilà. Gli animali nella «Commedia» di Dante, Ravenna, Longo, 2019, pp. 180-182 e 210-213.

17. Come avvertiva già E. Auerbach, Passi della «Commedia» dantesca illustrati da testi figurali. I: "Aquila volans ad escam» [1946], trad. it. in Studi su Dante, Milano, Feltrinelli, 1963, pp. 243-247, a p. 246: «Non è però certo che quanto si riferisce all'aquila si riferisca anche a Lucia; tenderei anzi a credere che il sogno profetico abbia implicazioni più complesse dell'intervento di Lucia». 
18. Segnalo che è stata da alcuni identificata in Lucia anche la «donna [...] santa e presta» che appare nel secondo sogno purgatoriale di Dante e sollecita Virgilio a svelare le brutture della «femmina balba» (Purg. XIX, 26). Per una tale ipotesi, che richiederebbe una lunga discussione ma che mi pare comunque priva di sufficienti riscontri testuali, si vedano per esempio i commenti ad loc. di L. Pietrobono, M. Porena e D. Mattalia (tutti consultabili sul Dartmouth Dante Project).

19. Camilla era stata citata anche nel I canto, nella rassegna dei giovani virgiliani morti nelle guerre del Lazio, dove è definita «vergine», come nel testo virgiliano (Inf. I, 107). Il padre, infatti, per salvarla dai nemici che li inseguivano, invocò l'aiuto di Diana promettendo alla dea, in cambio della salvezza, di consacrarle la fanciulla, che crebbe perciò votata alla verginità. Nell'Eneide è definita «decus Italiae virgo» (XI, 508); poi virgo ai vv. 604 («virginis [...] Camillae»), 664, 676, 718, 762, 778, 791; 808 (e si vedano anche i vv.583-584 e 804). In Dante una tale indicazione, che pure ha il senso latino di 'fanciulla', partecipa forse anche al processo di risemantizzazione in senso cristiano attivo per altre espressioni virgiliane.

20. Su Giacobbe, cfr. Gn 25, 19-35, 27. Per altri importanti riferimenti a Giacobbe nella Commedia: sulla differenza e rivalità con Esaù, cfr. Par. VIII, 130-132 e XXXII, 68-69; in riferimento al sogno in cui Giacobbe vide una scala che dalla terra andava verso il cielo, cfr. Par. XXII, 70-72.

21. Sul concetto di parodia sacra e per la sua applicazione ad alcuni passaggi dell'Inferno, cfr. per esempio G. Gorni, Parodia e Scrittura in Dante, in G. Barblan (a cura di), Dante e la Bibbia, Firenze, Olschki, 1988, pp. 323-340; E. Ardissino, Parodie liturgiche nell'«Inferno», «Annali di Italianistica», vol. XXV, 2007, pp. 217-232. F. Zanini, «Simulacra gentium argentum et aurum». Parodia sacra $e$ polemica anticlericale nell'«Inferno», «L'Alighieri», a. LIII, n.s. 39, 2012, pp.133-147; Id., Parodie liturgiche nell'«Inferno»: nota sulla Confessione, in G. Ledda (a cura di), Preghiera e liturgia nella «Commedia», Ravenna, Centro Dantesco dei Frati Minori Conventuali, 2013, pp. 155-190; G. Ledda, La Bibbia di Dante, Torino, Claudiana, 2015, pp. 29-43; P. Frare, Forme del male. Parodia e antitesi nell' «Inferno» di Dante, in M. Ballarini, G. Frasso e F. Spera (a cura di), Peccato, penitenza e santità nella «Commedia», Roma / Milano, Bulzoni / Biblioteca Ambrosiana, 2016, pp. 81-98; F. Zanini, «Cui non si convenia più dolci salmi». Osservazioni sulla parodia sacra nell' «Inferno» dantesco, in A. Campana e F. Giunta (a cura di), Natura Società Letteratura, Atti del XXII Congresso dell'ADI-Associazione degli Italianisti (Bologna, 13-15 settembre 2018), Roma, ADI editore, 2020, solo online. In particolare, sulla parodia agiografica nell'Inferno, cfr. G. Ledda, Poesia e agiografia nella «Commedia», cit., pp. 231-243.

22. Cfr. G. Varanini, Dante e Lucca, in G. Di Pino (a cura di), Dante e le città dell'esilio, Ravenna, Longo, 1989, pp. 91-114, a pp. 99-100. Il dato è ricordato da molti commentatori e valorizzato, fra gli altri, nel tessuto retorico complessivo del canto da A. Battistini, L'inabissamento nella «tenace pece», in Id., La retorica della salvezza. Studi danteschi, Bologna, Il Mulino, 2016, pp. 89-114, a p. 98.

23. Cfr. ancora G. Varanini, Dante e Lucca, cit., pp.100-102; A. Battistini, L'inabissamento nella «tenace pece», cit., pp. 102-103. Inoltre, E. Artale, Dal «fiorin d'oro d'amor» al «maladetto fiore», «Semicerchio», vol. XII, 1995, pp. 33-41.

24. Su cui cfr., anche per ulteriori riferimenti bibliografici, N. J. Vickers, "Seeing is Believing»: Gregory, Trajan and Dante's Art, «Dante Studies», $\mathrm{n}^{\circ}$ 101, 1983, pp. 67-85; M. C. Storini, Dante e la prosa italiana antica: una lettura dell'esempio di Traiano («Purgatorio», X, vv. 76-93), «Linguistica e Letteratura», vol. XXXVII, 2012, pp.9-38; V. Montemaggi, Dante and Gregory the Great, in C.E. Honess e M. Treherne (a cura di), Reviewing Dante's Theology, Oxford / Bern / Berlin, Lang, 2013, vol. I, pp. 209-262.

25. Iacopo da Varazze, Legenda aurea, cap. III, par. 17-27 (ed. cit., p. 38).

26. Iacopo da Varazze, Legenda aurea, cap. III, par. 11 (ed. cit., p. 38).

27. Iacopo da Varazze, Legenda aurea, cap. III, par. 18 (ed. cit., p. 39).

28. Cfr. in proposito Erminia Ardissino, «Pregar pur ch'altri prieghi» («Purg.»VI, 26). Richieste di suffragio nel «Purgatorio», in Preghiera e liturgia nella "Commedia», Città del Vaticano, Libreria Editrice Vaticana, 2009, pp. 45-66. 
29. Cfr. Purg. IV, 133-135; V, 70-72 e 88-90; VI, 1-12 e 25-48; VIII, 70-72; XI, 31-36; XIII, 124-129; XVI, 50-51; XIX, 91-96 e 142-145; XXIII, 76-90.

30. Cfr. in proposito le belle osservazioni di A. M. Chiavacci Leonardi, Le beatitudini e la struttura del «Purgatorio», cit.

31. Per una prima informazione rimando alla voce Matelda dell'Enciclopedia dantesca, firmata da Fiorenzo Forti.

32. Su queste due figure si vedano ora gli interventi di A. Pegoretti, La suora mancata: Piccarda, e F. Meier, L'imperatrice: Costanza, entrambi in F. Suitner (a cura di), Nel Duecento di Dante: i personaggi, Firenze, Le Lettere, 2020, rispettivamente pp. 19-37 e 59-82.

33. Sulla pericolosità del porsi tali domande restano a mio avviso efficaci le avvertenze di T. Barolini, The Undivine «Comedy». Detheologizing Dante, Princeton, Princeton University Press, 1992, pp. 3-20.

34. Cfr. L. Di Fonzo, Chiara d'Assisi, santa, in Enciclopedia Dantesca, Roma, Istituto della Enciclopedia Italiana, 1970-1978.

35. Per una prima informazione su Chiara, cfr. anche C. Frugoni, Una solitudine abitata, Bari, Laterza, 2006; M. Guida, Una leggenda in cerca d'autore. La Vita di Santa Chiara. Studio delle fonti e sinossi intertestuale, Bruxelles, Société des Bollandistes, 2010.

36. Tommaso da Celano, Vita Beati Francisci, cap. VIII, par. 18 (ed. a cura di C. Leonardi, in La letteratura Francescana, a cura di C. Leonardi, vol. II: Le Vite antiche di san Francesco, Milano, Fondazione Lorenzo Valla-Arnoldo Mondadori, 2005, p. 60).

37. Tommaso da Celano, Vita Beati Francisci, cap. VIII, par. 19 (ed. cit., pp. 60-62).

38. Sulla metafora nuziale e sul tema amoroso nel canto III, si vedano le belle osservazioni di E. Ardissino, Nuclei tematici nel canto di Piccarda («Paradiso» III), «L'Alighieri», a. LIII, n.s. 39, 2012, pp. 109-121, specialmente pp.114-116. Rilevanti anche le considerazioni sul tema dell'abito monacale: ivi, pp. 118-120. La connessione fra l'uso della metafora nuziale per santa Chiara e quello che sarà poi fatto per san Francesco nel canto XI è segnalata con particolare enfasi da N. R. Havely, Dante and the Franciscans. Poverty and the Papacy in the "Commedia», Cambridge, Cambridge University Press, 2004, p. 130. Per le connessioni della metafora nuziale usata da Piccarda con il linguaggio amoroso proprio della mistica, cfr. invece L. Battaglia Ricci, Canto III. "Ne' mirabili aspetti / vostri risplende non so che divino». Nel cielo della Luna, davanti ai primi beati, in E. Malato e A. Mazzucchi (a cura di), Lectura Dantis Romana. Cento anni per cento canti, vol. III: «Paradiso», Roma, Salerno Editrice, 2016, pp. 85-110, a pp. 102-104. Sulle radici bibliche della metafora nuziale usata nei canti del cielo del Sole a partire dal Cantico dei Cantici e dalla sua esegesi medievale, cfr., anche per altri riferimenti bibliografici, P. Nasti, The Amorous Bride and Her Lovers: Images of the Church in the Heaven of the Sun, in P. Acquaviva e J. Petrie (a cura di), Dante and the Church. Literary and Historical Essays, Dublin, Four Court Press, 2007, pp. 93-125; Ead., «Caritas» and Ecclesiology in Dante's Heaven of the Sun, in V. Montemaggi e M. Treherne (a cura di), Dante's «Commedia». Theology as Poetry, Notre Dame, Notre Dame University Press, pp. 210-244.

39. Bonaventura da Bagnoregio, Legenda maior sancti Francisci, cap. IV, par. 6, 3-4 (ed. a cura di C. Leonardi, in La letteratura Francescana, a cura di C. Leonardi, vol. IV, Milano, Fondazione Lorenzo Valla-Arnoldo Mondadori, 2013, p. 82).

40. Bonaventura da Bagnoregio, Legenda maior sancti Francisci, cap. XV, par. 5, 3 (ed. a cura di C. Leonardi, cit., p. 250).

41. Cfr. L. Di Fonzo, Chiara d'Assisi, santa, cit.

42. Per diverse interpretazioni degli aspetti relativi alla problematica presenza/assenza della tematica amorosa nei canti del cielo di Venere, mi limito a rimandare a L. Pertile, La punta del disio. Semantica del desiderio nella «Commedia», Fiesole, Cadmo, 2005, pp. 235-256; D. Pirovano, Dante e il vero amore. Tre studi danteschi, Roma, Salerno Editrice, 2009, pp. 33-89; S. Gentili, Canti VIII-IX. L'arco di Cupido e la freccia di Aristotele, in T. Montorfano (a cura di), Esperimenti danteschi. «Paradiso» 2010, Genova / Milano, Marietti 1820, 2010, pp. 87-112. 
43. Su Cunizza rimando al fondamentale volume di V. L. Puccetti, Fuga in Paradiso. Storia intertestuale di Cunizza da Romano, Ravenna, Longo, 2010. Per un intervento recente, cfr. R. Norbedo, Dal mito alla storia. Ancora su Cunizza nel Cielo di Venere («Paradiso»VIII e IX), «Rivista di Letteratura Italiana», vol. XXXVIII, $\mathrm{n}^{\circ}$ 2, 2020, pp. 27-37.

44. Su Raab, oltre alle lecturae del canto già citate, cfr. in particolare D. Glenn, Dante's Reforming Mission and Women in the "Comedy", Leicester, Troubador Publishing, 2008, pp. 99-121; P.S. Hawkins, Dante's Rahab, «Modern Language Notes», vol. CXXIV, ${ }^{\circ}$ 5, 2009, pp. 70-80.

\section{RIASSUNTI}

L'articolo passa in rassegna le sante e le beate citate nella Commedia di Dante. Un rilievo strutturale molto forte hanno, oltre ovviamente a Beatrice, la Vergine Maria e santa Lucia. Dopo aver promosso il soccorso di Dante, Maria è molto citata nel Purgatorio, sia come soccorritrice a cui rivolgere preghiere sia come esempio supremo di ogni virtù. Lucia continua a svolgere invece un diretto ruolo strutturale. Altre figure femminili sono presenti, con varie modalità e funzioni, negli esempi del Purgatorio. Nel Paradiso le donne beate sono incontrate solo nel primo e nel terzo cielo, ma poi sono numerose e significative le presenze femminili nell'Empireo. Inoltre assumono ancora un valore speciale, come a incorniciare la cantica, le figure di santa Chiara e di santa Lucia.

L'article passe en revue les saintes et bienheureuses mentionnées dans la Divine Comédie de Dante. En plus de Béatrice, bien sûr, la Vierge Marie et sainte Lucie ont une importance structurelle très forte. Après avoir favorisé le salut de Dante, Marie est souvent mentionnée dans le Purgatoire, à la fois comme un sauveur auquel on adresse des prières et comme un exemple suprême de toutes les vertus. Lucie, en revanche, continue à jouer un rôle structurel d'intermédiaire entre la Vierge et Béatrice. D'autres figures féminines sont présentes, avec des modalités et des fonctions diverses, dans les exemples du Purgatoire. Au Paradis, les femmes bénies ne se trouvent que dans le premier et le troisième ciel, mais il y a ensuite des présences féminines nombreuses et significatives dans l'Empyrée. En outre, les figures de sainte Claire et de sainte Lucie prennent une valeur particulière, comme si elles encadraient tout le Paradis.

The article gives a survey of the holy and blessed women mentioned by Dante in his Comedy. Some women have strong structural functions: it is the case of Beatrice, the Virgin Mary, and Saint Lucy. Mary is the one who starts Dante's rescue, then she is frequently mentioned in the Purgatory, not only as the rescuer to which one can address prayers, but also as the supreme model of every virtue. Saint Lucy plays again a direct structural role. Some other female figures are mentioned, in various ways and functions, also in the examples of the Purgatory. In the Paradise, some blessed women appear to Dante only in the first and the third heaven. But later many significant female figures characterizes the Empyrean. Morevoer, a special importance is given to Saint Clare and Saint Lucy, who are mentioned in the beginning and in the end, as to frame the third canticle. 
INDICE

Mots-clés : Dante, femmes, sainteté féminine, hagiographie

Keywords : Dante, Women, Holy Women, hagiography

Parole chiave : Dante, donne, santità femminile, agiografia

\section{AUTORE \\ GIUSEPPE LEDDA}

Giuseppe Ledda è professore associato di Letteratura italiana all'Università di Bologna. Oltre che di numerosi saggi e articoli su volumi e riviste in Italia e all'estero, è autore di alcuni libri di argomento dantesco, fra cui La Bibbia di Dante, Torino, Claudiana, 2015; Leggere la Commedia, Bologna, Il Mulino, 2016; Il bestiario dell'aldilà. Gli animali nella Commedia di Dante, Ravenna, Longo, 2019. È condirettore della rivista «L'Alighieri» e membro del Comitato Direttivo della rivista «Studi Danteschi».

Giuseppe Ledda est professeur associé de littérature italienne à l'Université de Bologne. Outre de nombreux essais et articles parus dans des livres et des revues en Italie et à l'étranger, il est l'auteur de plusieurs ouvrages sur Dante, notamment La Bibbia di Dante, Turin, Claudiana, 2015 ; Leggere la Commedia, Bologne, Il Mulino, 2016 ; Il bestiario dell'aldilà. Gli animali nella Commedia di Dante, Ravenne, Longo, 2019. Il est co-directeur de la revue L'Alighieri et membre du comité directeur de la revue Studi Danteschi. giuseppe.ledda@unibo.it 